\title{
Impact of Multi-Terminal HVDC Grids on Enhancing Dynamic Power Transfer Capability
}

\author{
Tatiana Mariano Lessa Assis, Senior Member, IEEE, Stefanie Kuenzel, Member, IEEE, and Bikash Chandra Pal, \\ Fellow, IEEE
}

\begin{abstract}
This paper proposes the exploitation of MultiTerminal HVDC grids to improve transfer capability in power systems. Multi-Terminal HVDC systems based on voltage source converters (VSC-MTDC) have been recognized as a promising alternative for the wind power integration. Under low wind scenarios, these grids originally dedicated for wind power transmission can be exploited as an additional interarea transmission path, providing extra dynamic security. The paper focuses on small-signal stability assessment, especially in poor damped oscillations associated with interarea modes. Simulations performed through a generic computational framework have shown that the high level of flexibility and controllability provided by voltage source converters can considerably improve the transfer capacity, while preserving adequate dynamic performance.
\end{abstract}

Index Terms - Transmission capability, security regions, VSCMTDC systems, dynamic security, wind power integration.

\section{INTRODUCTION}

$\mathrm{T}$ HE growing energy demand associated with environmental constraints and renewable generation technologies has brought new challenges to power system operators. Those challenges are also related to customers' requirements that include high power quality and high degree of reliability. The intermittency of renewable generation, especially of wind and solar plants, has a significant impact in system operation and planning [1].

Driven by various low carbon initiatives, the development of large wind farms has taken unprecedented priority in recent times [2]. With the best wind resource being over coastal water, offshore wind farms lead the development portfolios. Over larger distances, more than $100 \mathrm{~km}$ for high voltage

This work was supported in part by the Brazilian Federal Agency for Support and Evaluation of Graduate Education (CAPES), by the Rio de Janeiro State Foundation for Research Support (FAPERJ) and as part of the research project 'Stability and Control of Power Networks with Energy Storage (STABLE-NET)', which is funded by the RCUK's Energy Programme (contract no: EP/L014343/1).

T. M. L. Assis is with the Electrical Engineering Department of Federal University of Rio de Janeiro (COPPE/UFRJ), Rio de Janeiro, Brazil (e-mail: tatiana@dee.ufrj.br).

S. Kuenzel is with the Department of Electrical and Electronic Engineering, Imperial College London, London, U.K. (e-mail: stefanie.kuenzel06@imperial.ac.uk).

B. C. Pal is with the Department of Electrical and Electronic Engineering, Imperial College London, London, U.K. (e-mail: bcpal@ieee.org). levels and power rating, HVDC is the preferred transmission option [3].

Classical point-to-point HVDC transmission systems are well established for bulk DC power transmission and employ line-commutated converters (LCC) [4]. The advent of high power semiconductor switches with turn-on and turn-off capability has resulted in the development of voltage source converters (VSC). VSC-HVDC systems have important advantages over classic LCC technology, including bidirectional power transfer without polarity reversal, need for less filters, black start capability and space saving [5]. Moreover, VSC technology uses lighter and stronger cables, making them particularly attractive for offshore transmission [6]. For several wind farms located within close proximity, the DC multi-terminal approach through voltage source converters (VSC-MTDC) is the way forward [7][8]. The world's first VSC-MTDC grid has started operation in China in 2013 [9].

One of the formidable challenges in VSC-MTDC development lies on the protection system for DC network faults [10]-[12]. Nevertheless, several advances in developing adequate models and control strategies have already been made [13]-[19]. The authors in [13] have mathematically derived a general VSC-MTDC model valid for any topology of the DC grid. The interaction between multi-machine AC systems and a VSC-MTDC grid is discussed in reference [14]. The authors show that the cause of instability in certain cases can be attributed to the state variables related to the DC side. Reference [15] proposes an adaptive scheme for droop control strategy in VSC-MTDC systems and analyses the impact of using a variable droop scheme for autonomous power sharing during transient conditions. A methodology for control design of VSC-MTDC systems is proposed in [16], providing a criterion to select the DC droop control parameters. Kalcon and others [17] discuss the impact of VSC-MTDC control parameters on network stability taking into account of the small and large disturbances. The installation of classical power system stabilizer (PSS) in the onshore VSC stations for providing additional damping is investigated in [18]. Authors in [19] present a methodology to identify and analyse interaction modes between the converters in VSC-MTDC systems, largely influenced by their control parameters.

Despite several advances already made in developing adequate models and control strategies, the impact of VSCMTDC systems with regard to dynamic security has not yet been fully investigated. In this context, a crucial aspect is the interarea transfer capability that indicates how much power 
can be exchanged without compromising system security. Transfer capability is a key indicator for a competitive electric power market as well as for both planning and secure operation [20]. Topological alterations caused by unexpected contingencies or scheduled maintenances can drastically modify the amount of power that can be reliably exchanged [20]. In addition, the variability of renewable resources may change considerably the power flow in specific corridors, impacting positively or negatively the system operation. Specifically in low wind scenarios, one may observe a relief in the originally dedicated transmission paths, e.g. VSC-MTDC grids, which can be exploited in order to increase the exchange capacity.

This work proposes the exploitation of VSC-MTDC grids to improve transfer capability in power systems. The DC network is used as an additional interarea transmission path, especially under low wind scenarios. The idea is based on the concept of capacity factor, which is the ratio of the actual energy output over a period of time, to its potential output if it operated at full nameplate capacity over the same period. Reference [21] shows that the average capacity factor of Danish offshore wind farms is $41 \%$, which means that during $59 \%$ of the time, on average, the VSC-MTDC would be available for alternative use.

The work in this paper focuses on small-signal security as required during heavy transfers through long corridors, when any small changes lead to violating damping criteria. The proposed approach is evaluated through simulations in a twoarea power system using a generic computational framework.

The paper is organized as follows: Section II presents some background about transfer capability in power systems. The main characteristics of the computational framework developed for simultaneous analysis of AC and DC systems are described in Section III. Sections IV and V show the results obtained for a two-area system. Section VI presents the conclusions and Section VII is an appendix containing the test system parameters.

\section{TRANSFER CAPABILITY IN POWER SYSTEMS}

The open access to the transmission network in a competitive energy market characterized by different commercial transactions requires more secure and reliable transmission systems. In a way, the transfer capability is a measure of such security and reliability levels. It indicates how much power can be exchanged between different areas without violating a range of security criteria.

Traditionally, because of computational time limitations, especially in the on-line environment, the transfer capability was determined based only on static security assessment. In this context, voltage and thermal limits are observed through load flow analysis, taking into account a list of credible contingencies. Linearized power flow [22] and continuation power flow [23] have been used for this purpose.

It is recognized that transfer capability determination must take into account the system dynamic performance.
Therefore, accurate limits should be obtained based on full dynamic security assessment (DSA), where different aspects are observed, including transient, voltage and small-signal stability [24]. When two or more dynamic aspects are to be considered simultaneously in a security evaluation, conflicting objectives may be observed [25]. In this work, as the focus is on the interarea power transfer stability, only smallsignal security is considered besides the static evaluation.

Transfer capability in power systems can be analyzed in several ways. Two relevant aspects are the transmission capability through a specific transmission corridor and the security regions determination. Those aspects are discussed in the next sections.

\section{A. Transmission Capability through Specific Corridors}

There are different methodologies to compute the transmission capability considering load and generation changes. In this work, the system load is maintained constant during the whole process and, once the transmission path is established, the generation in the exporter area is increased while the generation in the importer area is decreased by the same amount. For a given exchange variation $(\Delta E)$, the power change $\left(\Delta P_{i}\right)$ in generator $i$ is determined by:

$$
\Delta P_{i}=\Delta E \times \frac{P_{i}}{\sum_{i=1}^{N_{A}} P_{i}}
$$

where $P_{i}$ is the prior output power of generator $i$ and $N_{A}$ is the number of generators within the area considered (exporter or importer). One should note that $\Delta P_{i}$ is positive in the exporter area and negative in the importer area. Moreover, $\Delta P_{i}$ should be obtained keeping the maximum capacity of each generator in consideration.

For each dispatch scenario, which corresponds to an exchange value, static and dynamic security assessments are performed based on pre-established criteria. If none of the criteria is violated, the system is considered secure and an additional redispatch is applied, using (1), in order to increase the exchange. Instead, if at least one of the security criteria is violated, a step back in the redispatch is applied and a binary search is conducted until a safe value is obtained.

\section{B. Security Regions}

Another important transfer capability-related aspect is the development of security regions [24]. Security regions are graphics that relate the active generation in different areas to indicate safe redispatch configurations. This is especially important in on-line applications to show to the operator how the power transfers can be changed, preserving the system integrity.

The authors in [26] present a methodology for the construction of security regions. Although they take into account only static security aspects, the method presented can be extended for dynamic security analysis.

One common way to build security regions starts with the definition of three generation groups (Groups \#1, \#2 and \#3), 
where one works as a slack group and the power transfer between the other two groups is evaluated. This philosophy results in a three-dimensional graph that contains the security boundaries with respect to the power generated in each group. In order to simplify the visualization, it is convenient to plot a nomogram, which is an orthogonal projection onto one of the generation planes [26]. The application of nomograms in voltage security analysis is discussed in [27], that argues how this graphical tool can be useful for system operators.

Figure 1 illustrates a security nomogram, relating the power produced by two generation groups: Group \#1 and Group \#2. The third group (Group \#3) does not appear in the nomogram shown in Fig. 1 because the nomogram is just an orthogonal projection onto one of the generation planes, for instance, (Group \#1 x Group \#2). However, it should be emphasized that two additional nomograms (Group \#2 x Group \#3) and (Group \#3 x Group \#1) could be plotted and would be similar to the one illustrated in Fig. 1.

From the initial operation point, redispatch directions are defined. Each direction, as the one illustrated in Fig. 1, is established by an angle $(\theta)$ that determines the proportion of power to be considered in each group for a given amount to be redispatched. Once defined the redispatch direction $(\theta)$ and the total power to be redispatched $(\Delta R)$, the power change in Group \#1 $\left(\Delta G_{1}\right)$ and Group \#2 $\left(\Delta G_{2}\right)$ are given by:

$$
\left\{\begin{array}{l}
\Delta G_{1}=\Delta R \times \cos \theta \\
\Delta G_{2}=\Delta R \times \sin \theta
\end{array}\right.
$$

The power change $\left(\Delta P_{i}\right)$ of generator $i$ within each group is determined by:

$$
\Delta P_{i}=\Delta G_{1,2} \times \frac{P_{i}}{\sum_{i=1}^{N_{G}} P_{i}}
$$

where $P_{i}$ is the prior output power of generator $i$ and $N_{G}$ is the number of generators within the group considered. Note that $\Delta P_{i}$ may assume positive or negative values depending on the redispatch direction. Moreover, $\Delta P_{i}$ should be computed while respecting the maximum capacity of each generator.

The third group (Group \#3, not shown in Fig. 1) works as a slack group, so the power balance can be reached as the system load is kept constant along the nomogram construction. For example, considering points in the first quadrant, where both Groups \#1 and \#2 have their generations increased, the power production in Group \#3 has to be reduced.

For each power change step, the system security is evaluated. The evaluation can include static and dynamic security. If a criterion is violated, a step back in the redispatch is applied and a binary search is conducted until the limit is reached. Note that for some directions, the power limit can be imposed by the generation capacity of one group, as exemplified by the squares points in Fig. 1.

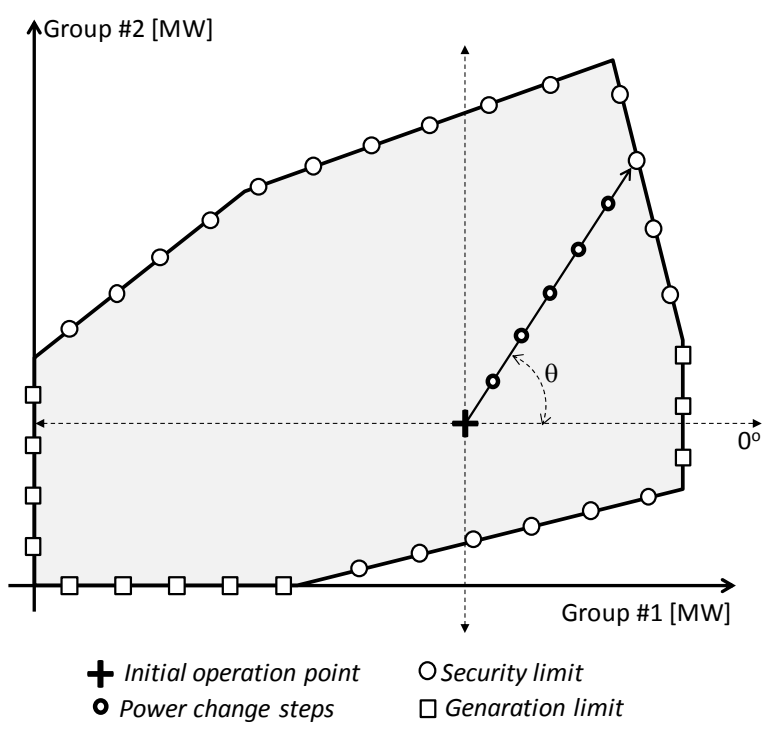

Fig. 1. Security region in a nomogram

For choosing the number of directions to be considered, one should balance the required processing time as well as the desired accuracy of the security boundary.

\section{MODELLING AND COMPUTATION FRAMEWORK}

The full understanding and analysis of integration of HVDC technology and wind generation into existing power systems require a generic model that represents all relevant components in a common framework. A generic modelling framework for small-signal stability studies and control design has been developed [28] and is used in this work to perform the simulations. This section provides overall characteristics about this framework, which is implemented in Matlab/Simulink ${ }^{\circledR}$. The modelling includes dynamic representation of synchronous generators, AC networks, converters, DC links and wind farms along with their associated controls.

The schematic representation of the simulation framework is shown in Fig. 2, where:

- $I_{\text {off }}$ is the vector of offshore currents leaving wind farms;

- $I_{c_{-} \text {off }}$ is the vector of offshore converters currents;

- $I_{o n}$ is the vector of onshore currents leaving wind farms;

- $V_{c_{-} \text {off }}$ is the vector of offshore converters voltages;

- $V_{\text {off }}$ is the vector of offshore voltages at winds farms;

- $V_{o n}$ is the vector of onshore voltages at wind farms;

- $\omega$ is the system frequency.

The block Main AC grid contains the model regarding the onshore AC network. The Synchronous gener ator s block represents all generators apart from wind. The Wind plants are represented by their own model, where some may be located off the main AC grid (offshore) and others onshore. The offshore wind plants are connected to an offshore AC network, represented by the block named off shore AC island, which also includes the AC side of the offshore converter stations. 


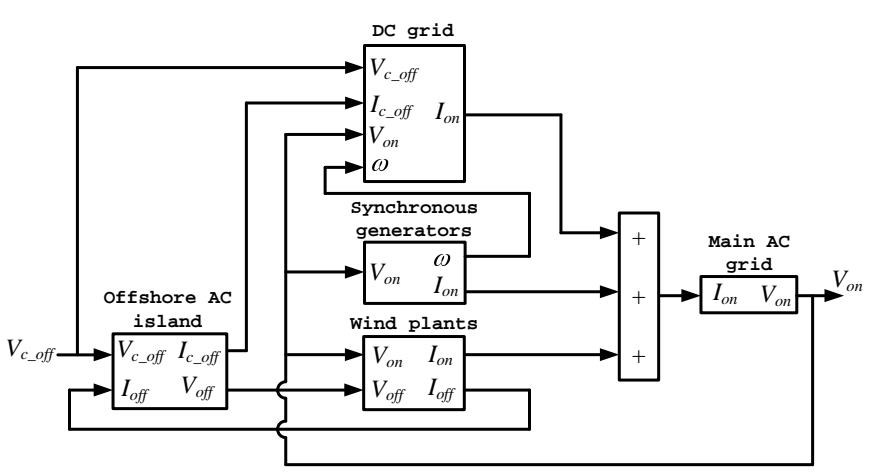

Fig. 2. Schematic representation of the simulation framework

The DC grid block models the interconnection of the offshore AC islands and the main AC grid. This block includes the section from the AC system bus to the AC converter bus. Hence, the interface voltages and currents for the DC grid block shown in Fig. 2 are AC quantities.

Using this generic framework, the DC grid topology, number of offshore AC grids, the main AC system and location and number of generators (synchronous and wind) can be chosen and altered easily, to allow the study of different expansion planning alternatives for such a system.

The modelling of the main AC grid, synchronous machines and associated controls are well established in the literature [29]. The next sections provide comments about the offshore AC grids, the wind farms and their control as well as the VSC-MTDC control. The initialization process of the entire model is also discussed.

\section{A. Offshore AC Grids}

As shown in Fig. 2, each offshore wind farm is connected to the DC grid via its own AC network, defining a wind generation (WG) island. The frequency of each WG island is determined by the power electronic converter. The power balance in each WG island is maintained by the converter's continuous regulation. This can be ensured by having the offshore converters regulating both voltage magnitude and phase angle at the converter bus.

The converter switching frequency depends on the VSC topology. The first generations were based on two- or threelevel converters that operate with fast switching $(1-2 \mathrm{kHz})$ [30]. Recent technology based on modular multilevel converters (MMC) can work with reduced switching frequency (few hundreds of Hz) [31]. However, in both cases, the converter voltage does not change instantaneously with changes in the reference value. Therefore, a delay between the control signal and the response from the converter exists and it is included in the model to account for the converter switching.

Wind farms located offshore are set to control reactive power. The wind turbine model provides a current output, according to terminal voltage, as illustrated in Fig. 2.

\section{B. Wind Farms and Controls}

Modelling the dynamic behaviour of wind farms is a challenging task as it depends on the exact turbine types, wind farm layout and wind farm cables [32]. When the behaviour of individual generators is of interest, the turbine model should contain a representation of the mechanical and electrical system. For system level studies a generic wind park model that is tuned and verified against measurements may be appropriate.

The developed framework is able to use the generic model and offshore network equivalent circuits as described in reference [33]. This generic model can be adopted to represent different types of wind generators, including doubly-fed induction generators and the full converter technology. Details about the specifications as well as the control characteristics of this generic model can be found in reference [33].

Although the developed framework is ready to use a generic wind power plant model and offshore network equivalent circuits, in the simulations presented in Section IV, the equivalent wind/offshore network is represented by a power injection. As the focus of the paper is on the interarea oscillations, this assumption does not bring drawbacks to the performed analysis.

\section{VSC-MTDC Control}

VSC-MTDC grid model adopted here is described in detail in reference [13]. It encompasses the converter $\mathrm{AC}$ buses and includes the dynamics of the DC grid. A VSC converter has two degrees of freedom: the angle and magnitude of the converter voltage, which can be used to independently control active and reactive power. Conventional proportional integral (PI) regulators are used to explore the two degrees of freedom provided by the converter.

The converter stations at the offshore side control the AC voltage magnitude and angle through fast converter control. The current injected into the converter bus is determined from the wind power plant model and offshore AC grid model (Fig. 2).

One converter station onshore is the DC slack converter station. It controls the DC voltage at its terminal and thus provides a voltage reference for the DC grid. Any mismatch between injected power, power drawn and losses will be accommodated by this bus. Offshore converter AC voltages are fixed and offshore currents are determined by the wind power models together with offshore AC island models.

\section{System Initialization}

Initial conditions, found via the solution of the AC-DC power flow, are provided at all integrator blocks. Since the offshore converter buses inject all the generated wind power into the DC grid, they are simulated as slack buses of the offshore WG islands. The DC slack bus can be at any of the onshore converter stations.

The power flow solution of all AC networks (the main grid plus the WG islands) is computed in a single procedure by aggregating the AC network information in one admittance matrix, with several disjoint parts, where each part contains at least one slack bus. This is an extension to the work in [13], which focuses on cases with only one AC and DC system. 
The power flow solution of the whole network requires four iterative processes. The first three being carried out sequentially: AC power flow, DC power flow and DC slack converter adjustment. The fourth performs an overall iteration to eliminate interface errors.

\section{TRANSMISSION CAPABILITY COMPUTATION}

This section presents results regarding the transmission capability calculation in a two-area system. All simulations have been performed with the framework described in Section III.

\section{A. Transmission Capability in the Original System}

Figure 3 shows the system one-line diagram in its original configuration (base case), i.e., without the VSC-MTDC grid. This system is a modified version of the two-area system presented in [29]. The generators are fully modelled, including the synchronous machines and the associated controls, i.e., automatic voltage regulators, governors and power system stabilizers. Section VII provides a complete set of parameters adopted in the test system [29]. The objective of the study is to calculate the maximum transmission capability from Area \#1 to Area \#2 through their interconnection lines.

The security criteria include safe voltage and thermal limits under steady-state operation (load flow analysis) and the minimum damping factor for small-signal stability assessment (SSA). One should note that other dynamic requirements, such as transient and voltage stability assessments should also be included for an entire DSA analysis. In this study, the focus is on SSA only because the transfer capability in the analysed system is restricted by poorly damped interarea oscillations.

The exchange from Area \#1 to Area \#2 is given by the power flow from Bus \#7 to Bus \#8, as indicated by the black arrows in Fig. 3. In order to increase such exchange, the generated power in Area \#1 (G1 and G2) is increased, while the generated power in Area \#2 (G3 and G4) is decreased by the same amount. These changes are made in steps and for each exchange level, steady-state and SSA analysis are performed. The power change $\left(\Delta P_{i}\right)$ in each generator is computed by (1), where the exchange variation $(\Delta E)$ adopted in each step is $50 \mathrm{MW}$.

Figure 4 shows the system evolution, presenting the interarea mode in the complex plane, as the exchange is increased. The initial exchange is $51 \mathrm{MW}$.

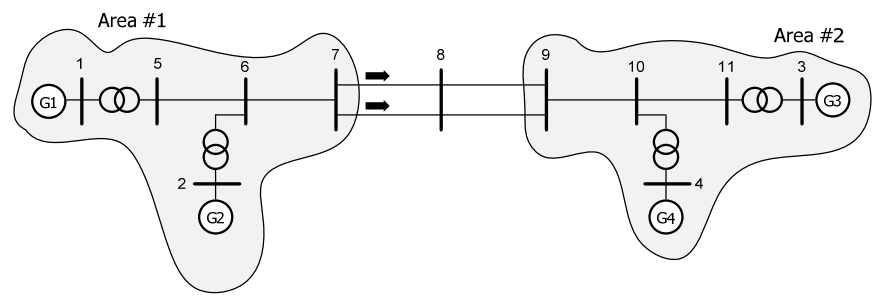

Fig. 3. Two-area: original system (base case)

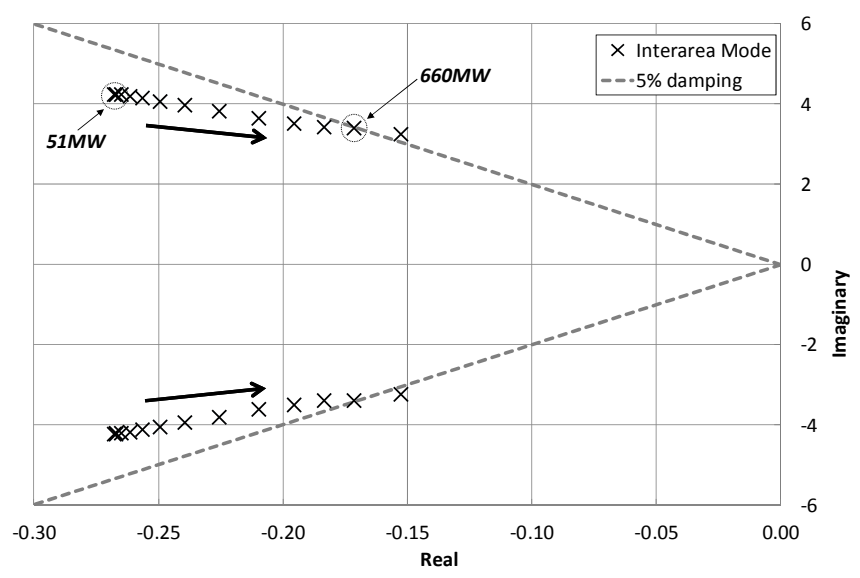

Fig. 4. Interarea mode for different exchanges: base case

The arrows shown in Fig. 4 indicate the direction of exchange increasing. Considering a minimal damping criterion of $5 \%$, the maximum transmission capability between Areas \#1 and \#2 is $660 \mathrm{MW}$. It is important to note that the minimum damping factor required is defined based on the operating characteristics of each system and damping factors less than the minimum required may cause the loss of synchronism among the generators [29].

\section{B. Transmission Capability with VSC-MTDC Grid}

The original system was modified in order to incorporate a VSC-MTDC grid for the integration of two offshore wind farms as illustrated in Fig. 5. G5 and G6 are able to deliver up to $400 \mathrm{MW}$ each, depending on the wind conditions. The equivalent wind/offshore network is represented by a power injection. As the focus of the paper is in the interarea oscillations, this is a reasonable assumption.

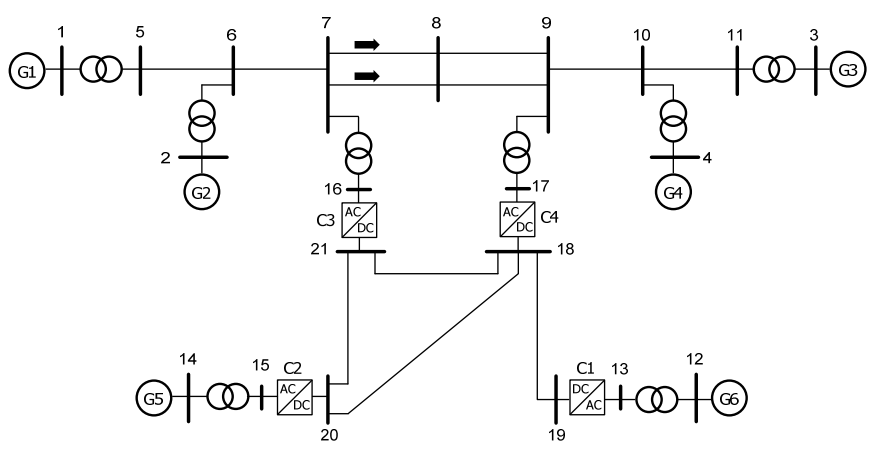

Fig. 5. Two-area system with VSC-MTDC grid

The VSC-MTDC system employed in the simulations is a symmetrically grounded, mono-polar four-terminal VSC system [6]. Fig. 6 illustrates the circuit of VSC grid with two converters, where $V_{d c}$ is the voltage potential from line to ground across a single capacitor. As indicated in Fig. 6, the model of the voltage source converter grid encompasses the dynamic behaviour of the connection between the system and converter AC buses. The model further includes the dynamics of the DC grid, with DC link capacitors, cable resistance 
and inductance. For simplicity, a two-terminal system is shown in Fig. 6. Regardless, the concept remains the same for a larger number of converter stations as considered in the test system shown in Fig. 5. At offshore converter stations, the converter voltage is fixed through the fast converter control and the modelling of dynamics from the converter transformer or phase reactor are incorporated in the offshore model, as can be seen in Fig. 2. All parameters adopted in the simulations are presented in Section VII [6].

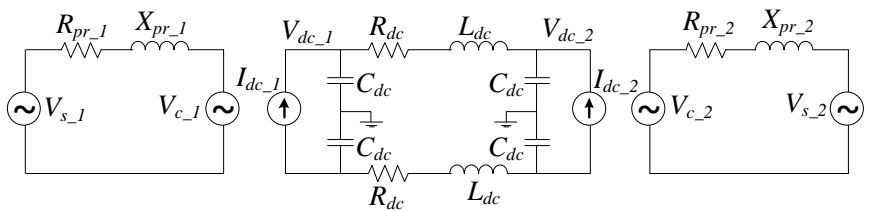

Fig. 6. Circuit of VSC grid with two converters

The offshore converters (C1 and C2 in Fig. 5) are in voltage control mode in the AC offshore side. Consequently, these converter stations produce a fixed voltage and hence pass all power coming from the offshore network directly into the DC grid. The onshore converter C3 operates in power control mode. Hence, active and reactive powers are specified at this station. In all simulations, a constant unit power factor was assumed. Finally, C4 is the DC slack converter. It controls the DC voltage at its terminal and thus provides a voltage reference for the DC grid. Any mismatch between injected power, power drawn and losses will be accounted for by this bus.

Initially, the power through converter C3 is set according to the wind generation at G5. It means that, except for the DC losses, the power produced by the wind generator G6 will pass through the DC slack converter C4.

Two scenarios are analysed. The first one is a low wind scenario where G5 and G6 produce only 10\% of their capacity (around $40 \mathrm{MW}$ each). Conversely, the second scenario assumes a high wind condition, with G5 and G6 at their full capacity (around $400 \mathrm{MW}$ each).

Figure 7 shows the interarea mode evolution for the low wind scenario. One can see the mode trajectory in the complex plane as the exchange is increased. The initial exchange is $29 \mathrm{MW}$ and the arrows shown in Fig. 7 indicate the direction of exchange increasing. Considering a minimum damping rate criterion of $5 \%$, the maximum transmission capability between Areas \#1 and \#2 is 722 MW.

Similar results are presented in Fig. 8, considering the high wind scenario. The interarea mode trajectory with increasing exchange is shown in the complex plane. In this case, the initial exchange is $28 \mathrm{MW}$ and the maximum transmission capability between Areas \#1 and \#2 is 799 MW for a $5 \%$ damping.

Significantly, the DC power flow does not change with the exchange increasing. This is justified because the wind

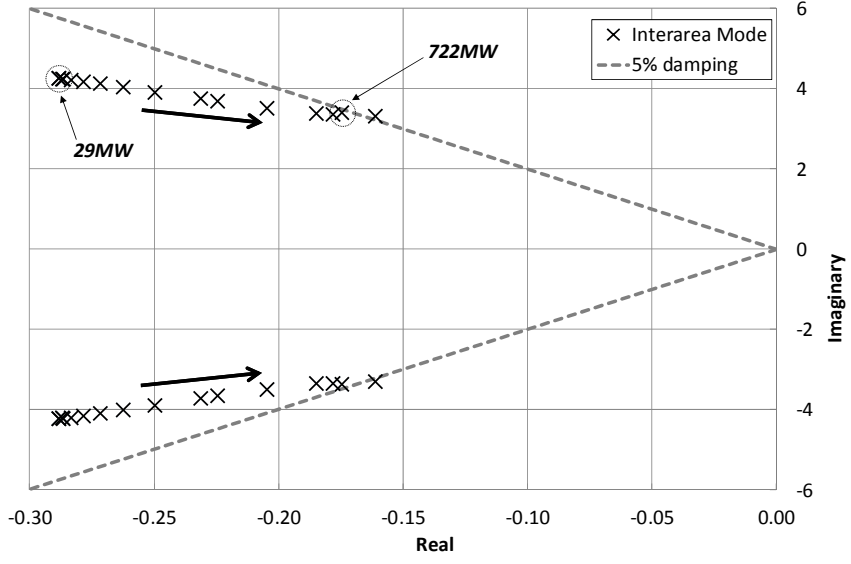

Fig. 7. Interarea mode for different exchanges: low wind scenario

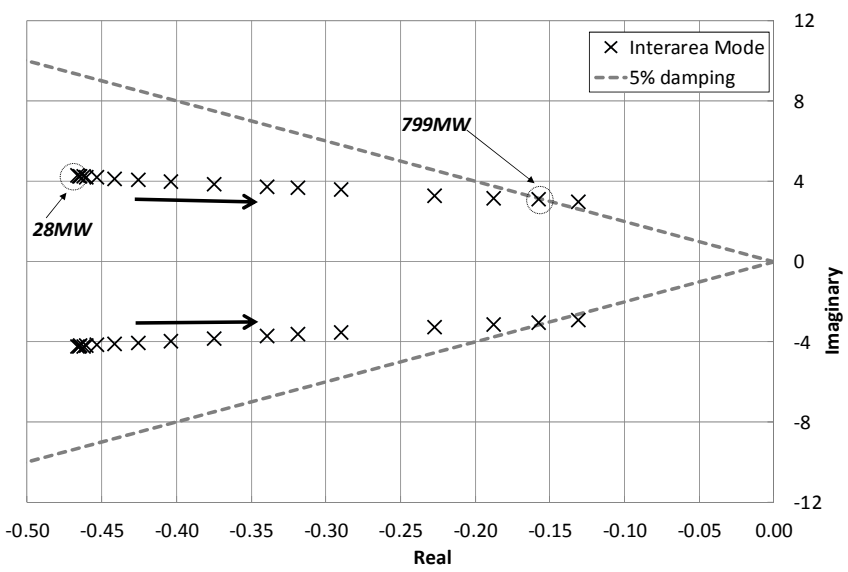

Fig. 8. Interarea mode for different exchanges: high wind scenario

generation (G5 and G6) is kept constant as well as the power set at converter C3. In this way, the power balance at converter C4 (slack converter) is the same as in all exchange conditions. This aspect can be verified in Table I that presents load flow results for the initial and the limiting operation points indicated in Fig. 7, considering the low wind scenario. Analogous results are observed in the high wind scenario. The sum of DC flows through lines \#21-18 and \#20-18 (Fig. 5) is low and the powers injected by converters C3 and C4 into the AC system are constant. It means that the exchange from Area \#1 to Area\#2 is given by the power flow from Bus \#7 to Bus \#8, as indicated by the black arrows in Fig. 5. As a result, the DC grid is not explored to improve the transmission capability.

TABLE I. LOAD FLOW RESULTS WITHOUT VSC-MTDC EXPLOITATION: LOW WIND SCENARIO

\begin{tabular}{c|c|c}
\hline $\begin{array}{c}\text { Power Flow } \\
\text { [MW] }\end{array}$ & $\begin{array}{c}\text { Initial Operation } \\
\text { Point }\end{array}$ & $\begin{array}{c}\text { Limiting Operation } \\
\text { Point }\end{array}$ \\
\hline Injection by C3 & 40.00 & 40.00 \\
\hline Injection by C4 & 40.89 & 40.89 \\
\hline Lines \#7-8 & 28.86 & 721.77 \\
\hline Line \#21-18 & -13.02 & -13.02 \\
\hline Line \#20-18 & 13.98 & 13.98 \\
\hline Exchange & 29.82 & 722.73 \\
\hline
\end{tabular}


The evolution of the interarea mode damping with respect to the exchange values is presented in Fig. 9. Results for the base case, low and high wind scenarios are shown as well as the minimum damping line. In all situations, the damping decreases as the exchange increases. However, in the high wind scenario, the initial damping level is higher (around 11\%).

This is expected as the high injection from the VSCMTDC grid ( 2 x $400 \mathrm{MW})$ allows the power reduction of onshore generators (G1 to G4), improving the interarea mode damping for the same exchange level. Evidently, for this test system, the higher the VSC-MTDC power injection, the higher the transmission capability between Areas \#1 and \#2, as the limiting factor is the interarea oscillation damping. Also, the base case (without VSC-MTDC) and the low wind condition present similar results because the VSC-MTDC grid is not being exploited for power transferring between Areas \#1 and \#2.

The presented results show that no advantage is taken from the VSC-MTDC grid, because the operation philosophy of the converters makes them to work as two HVDC links independent from each other.

\section{Exploring the VSC-MTDC Grid}

In order to improve the transmission capability, the VSCMTDC grid can be used as an additional path between Areas \#1 and \#2. This is especially interesting when low wind scenarios occur and the DC grid is idle. Figure 10 illustrates the concept of exploiting the VSC-MTDC grid to send exceeding power from Area \#1 to Area \#2. The transmission path now includes the DC lines between Areas \#1 and \#2.

In this situation, the power set in converter $\mathrm{C} 3$ will directly impact on the interarea mode damping. Table II shows the damping factor for different power settings (injected power) in converter C3. All cases consider low wind conditions where G4 and G5 are producing around 40 MW each. Negative values of injected power mean that part of the generation in Area \#1 is getting into the VSC-MTDC grid from converter C3 and returning to the onshore AC system (Area \#2) through converter $\mathrm{C} 4$.

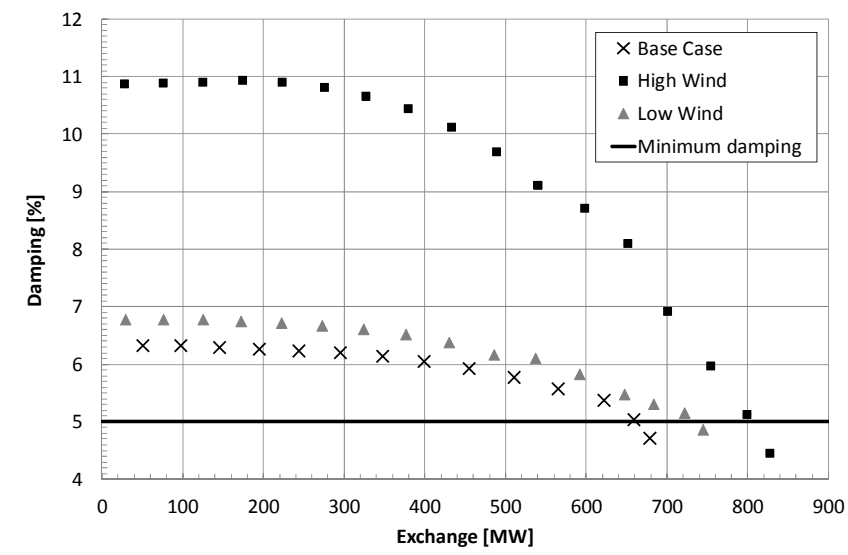

Fig. 9. Damping comparison for different exchanges

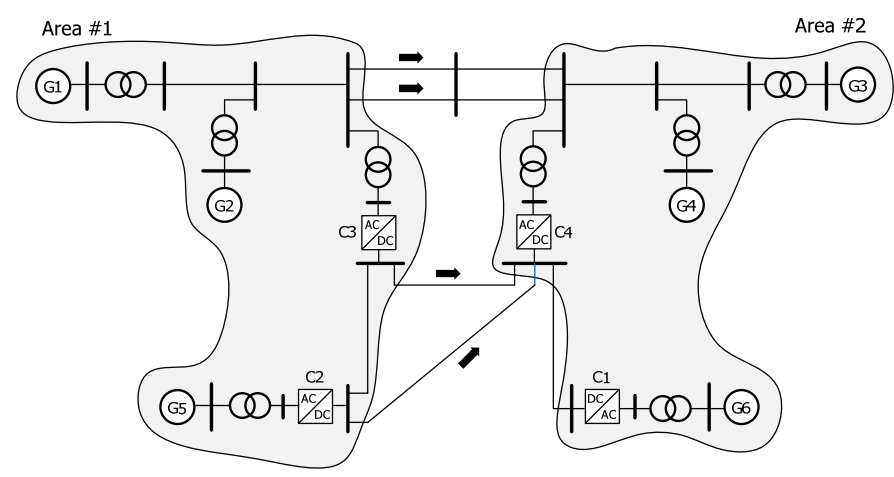

Fig. 10. Two-area system with VSC-MTDC grid exploitation

The exchange from Area \#1 to Area \#2 is practically constant in all cases presented in Table II. As a result, the damping increases as the power drained from the AC side of converter $\mathrm{C} 3$ increases and the power through the AC path is being reduced.

TABLE II. DAMPING FACTOR FOR FIXED EXCHANGES

\begin{tabular}{c|c|c}
\hline $\begin{array}{c}\text { Exchange } \\
{[\mathrm{MW}]}\end{array}$ & $\begin{array}{c}\text { Injected Power } \\
{[\mathrm{MW}]}\end{array}$ & $\begin{array}{c}\text { Damping } \\
{[\%]}\end{array}$ \\
\hline 746 & 40 & 4.86 \\
\hline 747 & 0 & 5.58 \\
\hline 748 & -40 & 6.13 \\
\hline 748 & -80 & 6.57 \\
\hline 749 & -120 & 6.93 \\
\hline 749 & -160 & 7.23 \\
\hline 749 & -200 & 7.49 \\
\hline 749 & -240 & 7.76 \\
\hline 749 & -280 & 7.93 \\
\hline 749 & -320 & 8.12 \\
\hline 749 & -360 & 8.25 \\
\hline 749 & -400 & 8.32 \\
\hline
\end{tabular}

Figure 11 shows the results of Table II. The point below the minimum damping line corresponds to the case where C3 power is set according to G4 production (40 MW). In this case, the $746 \mathrm{MW}$ exchange is not acceptable from the SSA point of view. Conversely, the same exchange level can be safely allowed if the VSC-MTDC grid is exploited.

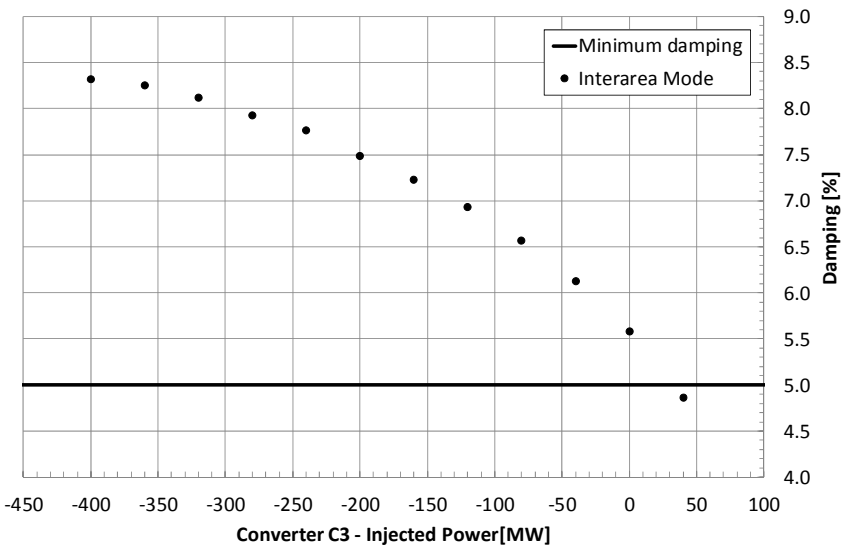

Fig. 11. Damping for different injected powers 
TABLE III. LOAD FLOW RESULTS WITH AND WITHOUT VSC-MTDC EXPLOITATION: LOW WIND SCENARIO

\begin{tabular}{c|c|c}
\hline $\begin{array}{c}\text { Power Flow } \\
\text { [MW] }\end{array}$ & $\begin{array}{c}\text { Without VSC-MTDC } \\
\text { Exploitation }\end{array}$ & $\begin{array}{c}\text { With VSC-MTDC } \\
\text { Exploitation }\end{array}$ \\
\hline Injection by C3 & 40.00 & -400.00 \\
\hline Injection by C4 & 40.89 & 476.14 \\
\hline Lines \#7-8 & 744.75 & 309.01 \\
\hline Line \#21-18 & -13.02 & 280.34 \\
\hline Line \#20-18 & 13.98 & 160.02 \\
\hline Exchange & 745.71 & 749.37 \\
\hline
\end{tabular}

Table III presents load flow results for the two cases highlighted in Table II. Although both cases in Table III have similar exchange values, when the DC grid is exploited, forcing the AC power flow to be deviated through converter $\mathrm{C} 3$, the DC interconnection lines (\#21-18 and \#20-18) became more loaded, relieving the AC path (\#7-8). If C3 is set to drain 400 MW from Area \#1, G1 and G2 could generate their maximum power ( $2 \times 900 \mathrm{MW})$, the exchange would be $827 \mathrm{MW}$ and the damping of the interarea mode would stay above the minimum required (8.19\%).

In order to illustrate the system dynamic performance, Fig. 12 compares the time-domain response for the two cases highlighted in Table II. A positive (negative) step of $1 \%$ is applied in the speed references of generators of Area \#1 (Area \#2) to excite the interarea mode. The speed deviations $(\Delta \omega)$ shown in Fig. 12 clearly indicate the damping differences pointed out in Table III (8.32\% vs 4.86\%). Moreover, the interarea mode can be easily identified as G1 and G2 oscillate against G3 and G4. One should remember that the exchange level is the same in both cases. Nevertheless, the injected power through converter C3 is $-400 \mathrm{MW}$ in case (a) and $+40 \mathrm{MW}$ in case (b).

Another important aspect related to the VSC-MTDC grid exploitation is a possible loss reduction. In general, losses in AC transmission are higher than the losses in the DC transmission, because of the presence of reactive power flow and the skin effect in AC systems. However, the change in losses will depend on the grid topology and it is an important point to be analysed if the DC grid is to be explored.

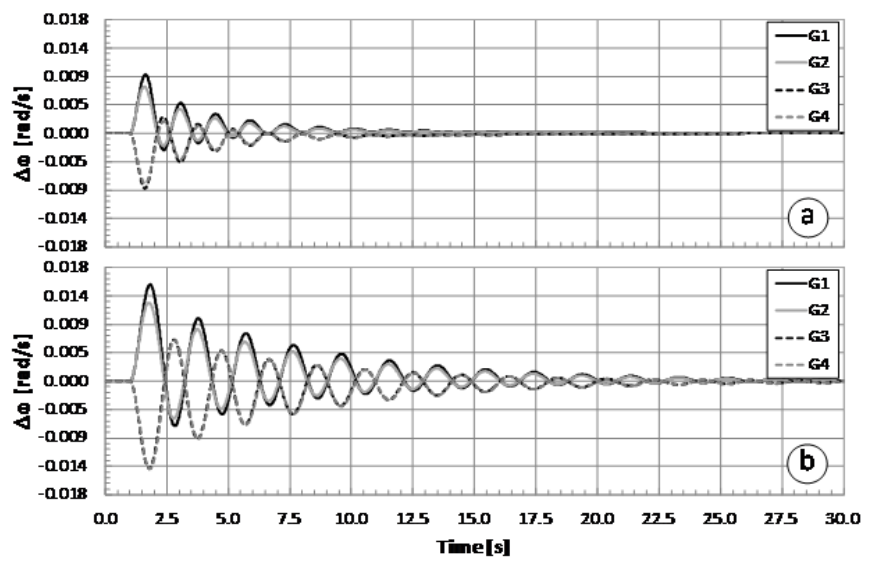

Fig. 12. Step response with (a) and without (b) VSC-MTDC grid exploitation
The total losses were computed in two cases highlighted in Table II. In the first case, when the VSC-MTDC system is not being explored (injected power through converter C3 is $40 \mathrm{MW}$ ), the total losses are $129 \mathrm{MW}$ (4.5\%). In the second case, when the VSC-MTDC system is being explored (drained power through converter C3 is $400 \mathrm{MW}$ ), the total losses are 68 MW (2.4\%). These values do not take into account the converters losses. Considering $3 \%$ of losses in the converters, which is a conservative estimate [30], the total losses would be $4.7 \%$ and $3.4 \%$ in the first and second case, respectively.

The grid topology also influences the damping improvement when the VSC-MTDC system is to be explored. However, the main influence is associated with the reduction of the power flow at the AC interconnection lines between Areas \#1 and \#2 (lines \#7-8). So, the central aspect to be considered is the point of the DC system connection with the AC one. For example, if the coupling buses were \#9 and \#11 instead of \#7 and \#9, the results would be significantly changed.

\section{SECURITY REgIONS DETERMINATION}

In order to further illustrate the possibility of exploring VSCMTDC grids to improve the transfer capability, this section shows the security regions computed for the two-area system described in Section IV. In this case, the generation groups are Group \#1 and Group \#2, composed by the generators of Area \#1 and \#2, respectively. Moreover, an additional slack group (Group \#3) was incorporated to the system, which is in charge of power balance. The slack group is essential to allow the exploration of the four quadrants in the plane (Group \#1 x Group \#2). So, once Groups \#1 and \#2 are defined, Group \#3 can be seen as the set of all generators in the system that belongs neither to Group \#1 nor to Group \#2. The test system was modified, supposing that the slack group is connected to Bus \#8, as illustrated in Fig. 13. For simplicity, it was modelled by a single power injection.

The initial operation point consists of the low wind scenario as described in Section IV. So, the offshore wind plants G5 and G6 produce $40 \mathrm{MW}$ each.

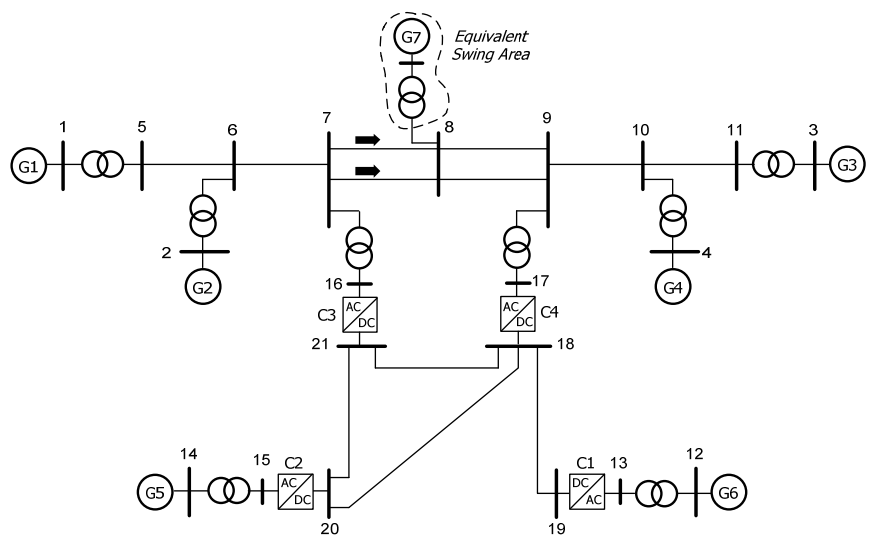

Fig. 13. Test system with an equivalent slack area 
For computing the security regions, 16 directions, as described in Section II, have been considered. It means that the angle $(\theta)$ in Fig. 1 assumes the values $22.5^{\circ}, 45^{\circ}, 67.5^{\circ}, \ldots$, $360^{\circ}$. For each direction, the amounts to be redispatched in Groups \#1 and \#2 are calculated by (2). Within each group, the generation change in each plant is done proportionally to the power produced in the prior operation point, according to (3), where the redispatched power $(\Delta R)$ adopted in every step is $50 \mathrm{MW}$. It is important to emphasize that the power balance must be satisfied and Group \#3 is in charge of this task. In this way, in each step of calculation, a generation set (Group \#1 x Group \#2 x Group \#3) is obtained and used to build the security region.

Successive redispatches are simulated and both static and dynamic security criteria are checked. When a criterion is violated, a step back in the redispatch is applied and a binary search is conducted until the limit is reached. The step back and the binary search are performed maintaining the same direction analysed.

The security regions are computed with and without the VSC-MTDC exploitation and the results are depicted in Fig. 14. It is evident that the security region is improved when the VSC-MTDC grid is exploited. It means that high power transfers are allowed without compromising system security.

Figure 15 shows the security nomogram relating the generation at Group \#1 and Group \#2. The axes limits correspond to the total generation capacity of each group $(2 \mathrm{x}$ $900 \mathrm{MW})$. In the initial operation point, the power produced by Group \#2 is close to its limit, so the increasing margin in such group is small. However, other multiple generation transfer directions can be explored.

When no VSC-MTDC grid exploitation is considered, the power flow injected by the VSC-MTDC grid into the AC system was kept constant, i.e., about $40 \mathrm{MW}$ through converters C3 and C4. Conversely, with the VSC-MTDC exploitation, the power through converter C3, which operates in power control mode, is changed in order to eliminate security criteria violations, allowing high power transfers.

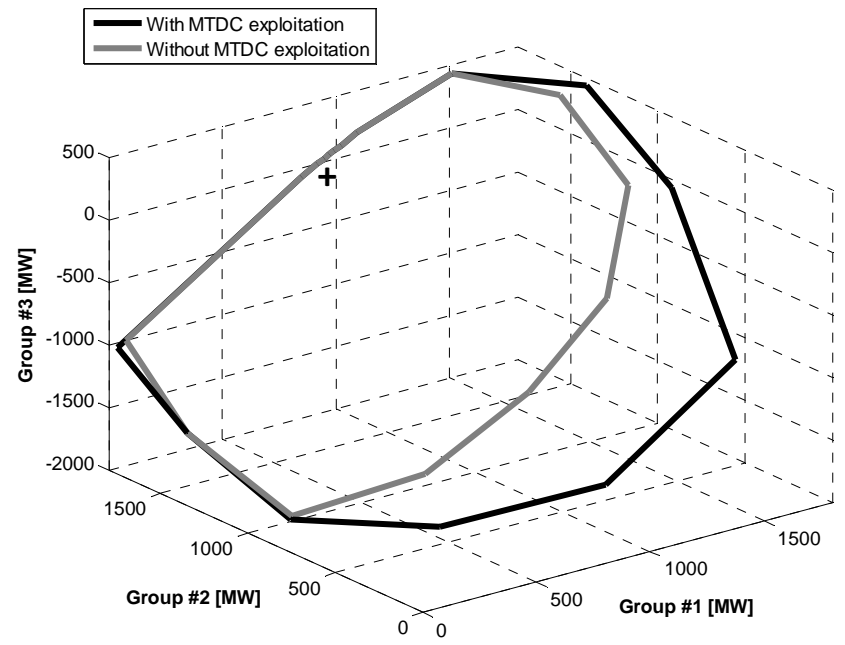

Fig. 14. Security region with and without VSC-MTDC grid exploitation

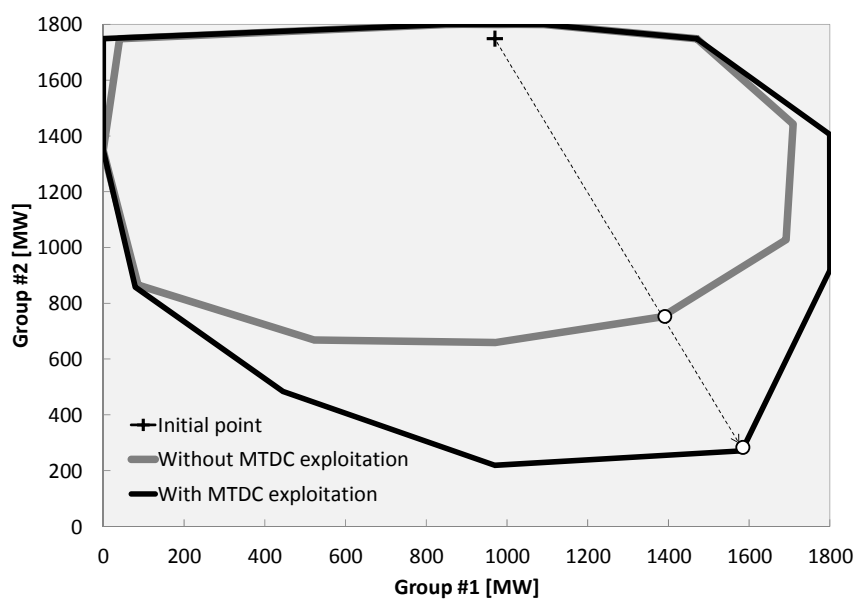

Fig. 15. Security nomogram with and without VSC-MTDC grid exploitation

For example, in the direction highlighted in Fig. 15, when the VSC-MTDC exploitation is not considered, the maximum amount of power that can be redispatched is $1080 \mathrm{MW}$, with an interarea mode damping of $5.57 \%$. From this point, an additional $10 \mathrm{MW}$ redispatch results in criterion violation (minimum 5\% damping). On the other hand, for the same direction, but exploring the VSC control capability, 1600 MW can be redispatched with a damping factor of $5.37 \%$. In this case, the power drained from the AC system by the converter C3 is $80 \mathrm{MW}$, which means that part of the AC power produced by Group \#1 is being diverted through the DC grid.

\section{CONCLUSIONS}

Multi-terminal HVDC systems based on voltage source converters (VSC-MTDC) are a promising alternative to connect several wind farms located within close proximity. Despite of huge challenges regarding the protection schemes of such systems, they are being widely discussed and investigated.

While wind farms have experienced exceptional development in recent times, the concern about power systems reliability increases. Transfer capability plays an important role in this scenario to support a competitive power market as well as secure operation.

This paper has investigated the exploitation of VSCMTDC grids to improve transfer capability, especially under low wind conditions. Grids originally dedicated for wind power transmission can be used as an additional interarea transmission path, providing extra dynamic security. The proposed idea was evaluated in a two-area system taking into account small-signal stability assessment. The results have shown that adequate power set at the DC network can ease the constraint of AC grid, allowing higher transfer with guaranteed security margin.

\section{APPENDIX}

This appendix provides a comprehensive set of parameters adopted in the test system described in Section IV. The parameters of the AC and DC systems have been obtained from references [29] and [6], respectively. 
The AC grid parameters are shown in Table IV, where all values are provided in a $100 \mathrm{MVA} / 230 \mathrm{kV}$ basis.

TABLE IV. AC GRID PARAMETERS

\begin{tabular}{c|c|c|c|c}
\hline From & To & $\mathbf{R}[\%]$ & $\mathbf{X}[\%]$ & $\mathbf{B}[\%]$ \\
\hline 1 & 5 & 0.000 & 1.667 & --- \\
\hline 2 & 6 & 0.000 & 1.667 & --- \\
\hline 4 & 10 & 0.000 & 1.667 & --- \\
\hline 3 & 11 & 0.000 & 1.667 & --- \\
\hline 5 & 6 & 0.250 & 2.500 & 4.375 \\
\hline 6 & 7 & 0.100 & 1.000 & 1.750 \\
\hline 7 & 8 & 1.100 & 11.000 & 19.250 \\
\hline 8 & 9 & 1.100 & 11.000 & 19.250 \\
\hline 9 & 10 & 0.100 & 1.000 & 1.750 \\
\hline 10 & 11 & 0.250 & 2.500 & 4.375 \\
\hline
\end{tabular}

Table V indicates the load and shunt capacitors values. The load model considers constant current and constant impedance characteristics for active and reactive components, respectively.

\begin{tabular}{c|c|c}
\multicolumn{3}{c}{ TABLE V. LOAD AND SHUNT CAPACITORS PARAMETERS } \\
\hline Bus & Load & Shunt Capacitor \\
\hline 7 & $(967+\mathrm{j} 100)[\mathrm{MVA}]$ & $200[\mathrm{Mvar}]$ \\
\hline 9 & $(1767+\mathrm{j} 100)[\mathrm{MVA}]$ & $350[\mathrm{Mvar}]$ \\
\hline
\end{tabular}

The parameters of the synchronous machines are presented in Table VI. All values are provided in the generators' basis (900 MVA, $20 \mathrm{kV}$ ).

TABLE VI. SYNCHRONOUS MACHINES PARAMETERS

\begin{tabular}{c|c|c}
\hline Parameter & G1 - G2 & G3 - G4 \\
\hline$X_{d}[\mathrm{pu}]$ & 1.8 & 1.8 \\
\hline$X_{q}[\mathrm{pu}]$ & 1.7 & 1.7 \\
\hline$X_{l}[\mathrm{pu}]$ & 0.2 & 0.2 \\
\hline$X^{\prime}{ }_{d}[\mathrm{pu}]$ & 0.3 & 0.3 \\
\hline$X^{\prime}{ }_{q}[\mathrm{pu}]$ & 0.55 & 0.55 \\
\hline$X^{\prime}{ }_{d}[\mathrm{pu}]$ & 0.25 & 0.25 \\
\hline$X^{\prime}{ }_{q}[\mathrm{pu}]$ & 0.25 & 0.25 \\
\hline$R_{a}[\mathrm{pu}]$ & 0.0025 & 0.0025 \\
\hline$T^{\prime}{ }_{d 0}[\mathrm{~s}]$ & 8.0 & 8.0 \\
\hline$T_{q 0}[\mathrm{~s}]$ & 0.4 & 0.4 \\
\hline${ }^{\prime}{ }_{d 0}[\mathrm{~s}]$ & 0.03 & 0.03 \\
\hline$T{ }_{q 0}[\mathrm{~s}]$ & 0.05 & 0.05 \\
\hline$H[\mathrm{MW} . \mathrm{s} / \mathrm{MVA}]$ & 6.5 & 6.175 \\
\hline
\end{tabular}

Figure 16 presents the governor model and parameters adopted in the simulations.

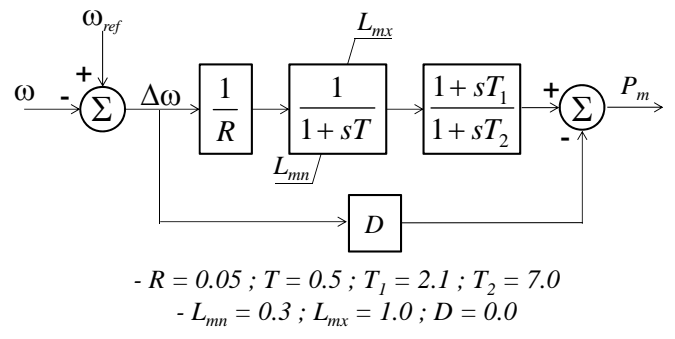

Fig. 16. Governor model and parameters
The automatic voltage regulator (AVR) and power system stabilizer (PSS) models and parameters are shown in Fig.17.

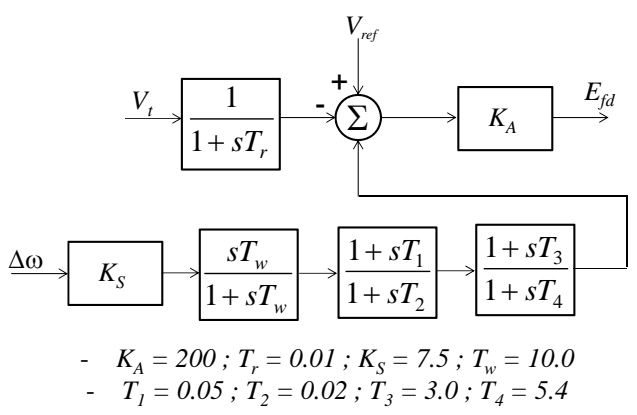

Fig. 17. AVR/PSS model and parameters

Table VII shows the VSC-MTDC grid parameters.

TABLE VII. VSC-MTDC GRID PARAMETERS

\begin{tabular}{c|c|c}
\hline Parameter & Description & Value \\
\hline$R_{d c}$ & Resistance of DC lines & $6[\Omega]$ \\
\hline$C_{d c}$ & Capacitance of CD lines & $0.4[\mathrm{mF}]$ \\
\hline$L_{d c}$ & Inductance of DC lines & $300[\mathrm{mH}]$ \\
\hline$V_{d c}$ & Nominal DC voltage & $\pm 350[\mathrm{kV}]$ \\
\hline$R_{p r}$ & Resistance of phase reactor & $0.07[\Omega]$ \\
\hline$X_{p r}$ & Reactance of phase reactor & $40[\mathrm{~m} \Omega]$ \\
\hline
\end{tabular}

\section{REFERENCES}

[1] L. E. Jones, "Renewable energy integration: Practical management of variability, uncertainty, and flexibility in power grids,” Academic Press, Elsevier Inc., 2014.

[2] Global Wind Energy Council, "Global wind report: Annual market update 2014,” March 2015. [Online]. Accessed April 2016. Available: http://goo.gl/AtsR5V.

[3] T. Ackermann N. B. Negra, J. Todorovic and L. Lazaridis, "Evaluation of electrical transmission concepts for large offshore wind farms," Proceeding of the Copenhagen Offshore Wind Conference, October 2005.

[4] J. Arrillaga, Y. H. Liu, and N. R. Watson, "Flexible power transmission: the HVDC options,” John Wiley, 2007.

[5] G. Li, C. Li and D. Van Hertem, "HVDC technology overview," in HVDC Grids: For Offshore and Supergrid of the Future, Chapter 3, Wiley-IEEE Press, pp. 45-78, 2016.

[6] N. Chaudhuri, B. Chaudhuri, R. Majumder and A. Yazdani, "Multi-terminal Direct-Current Grids: Modeling, Analysis, and Control," Wiley-IEEE Press, September 2014.

[7] T. Ackermann, "Transmission systems for offshore wind farms," IEEE Power Engineering Review, vol.22, no.12, pp.23-27, December 2002.

[8] P. Bresesti, W. L. Kling, R. L. Hendriks and R. Vailati, "HVDC connection of offshore wind farms to the transmission system,” IEEE Transactions on Energy Conversion, vol.22, no.1, pp.37-43, March 2007.

[9] "Rongxin Power Electronic (RXPE) commissions the world's first VSC multi-terminal HVDC project," 2013. [Online]. Accessed November 2015. Available: http://goo.gl/1sZhMo.

[10] T. Lianxiang and O. Boon-Teck, "Locating and isolating DC faults in multi-terminal DC systems," IEEE Transactions on Power Delivery, vol.22, no.3, pp.1877-1884, July 2007. 
[11] K. Kerf, K. Srivastava, M. Reza, D. Bekaert, S. Cole, D. Van Hertem, R. Belmans, "Wavelet-based protection strategy for DC faults in multi-terminal VSC HVDC systems," IET Generation, Transmission \& Distribution, vol.5, no.4, pp.496-503, April 2011.

[12] S. Azizi, M. Sanaye-Pasand, M. Abedini, A. Hassani, "A traveling-wave-based methodology for wide-area fault location in multiterminal DC Systems,” IEEE Transactions on Power Delivery, vol.29, no.6, pp.2552-2560, December 2014.

[13] S. Cole, J. Beerten and R. Belmans, "Generalized dynamic VSC MTDC model for power system stability studies,” IEEE Trans. on Power Systems, vol.25, no.3, pp.1655-1662, August 2010.

[14] N. R. Chaudhuri, R. Majumder, B. Chaudhuri, Jiuping Pan, "Stability analysis of VSC MTDC grids connected to multimachine AC systems," IEEE Transactions on Power Delivery , vol.26, no.4, pp.2774-2784, October 2011.

[15] N. R. Chaudhuri, B. Chaudhuri, "Adaptive droop control for effective power sharing in multi-terminal DC (MTDC) Grids,” IEEE Transactions on Power Systems, vol.28, no.1, pp.21-29, Feb. 2013.

[16] E. Prieto-Araujo, F. D. Bianchi, A. Junyent-Ferre and O. Gomis-Bellmunt, "Methodology for droop control dynamic analysis of multiterminal VSC-HVDC grids for offshore wind farms," IEEE Transactions on Power Delivery, vol. 26, no. 4, pp. 2476-2485, October 2011.

[17] G. O. Kalcon, G.O., G. P. Adam, O. Anaya-Lara, S. Lo and K. Uhlen, "Small-signal stability analysis of multi-terminal VSC-based DC transmission systems," IEEE Transactions on Power Systems, vol.27, no.4, pp.1818-1830, November 2012.

[18] F. O. Resende, M. H. Vasconcelos, J. A. Peças Lopes, J.A., "Simultaneous tuning of power system stabilizers installed in the VSC-based MTDC networks of large offshore wind farms," Proceedings of the 2014 Power Systems Computation Conference (PSCC), 18-22 August 2014.

[19] J. Beerten, S. D’Arco and J. A. Suul, "Identification and smallsignal analysis of interaction modes in VSC MTDC systems," IEEE Transactions on Power Delivery, vol. 31, no. 2, pp. 888-897, April 2016.

[20] NERC, “Available transfer capability definitions and determination,” North American Electric Reliability Corporation, June 1996.

[21] Energy Numbers, "Capacity factors at Danish offshore wind farms,” June 2016. [Online]. Accessed July 2016. Available: http://goo.gl/rwZaJB.

[22] S. Grijalva, P.W. Sauer and J.D. Weber, "Enhancement of Linear ATC calculations by the incorporation of reactive power flows”, IEEE Transactions Power Systems, vol. 18, no. 2, pp. 619624, May 2003.

[23] G.C. Ejebe, J. Tong, J.G. Waight, J.G. Frame, X. Wang and W. F. Tinney, “Available transfer capability calculations”, IEEE Trans. Power Systems, vol. 13, no. 4, pp. 1521-1527, November 1998.

[24] K. Morison, Lei Wang and P. Kundur, "Power System Security Assessment”, IEEE Power \& Energy Magazine, vol. 2, no. 5, September-October 2004, pp. 30-39.
[25] T. M. L. Assis, D. M. Falcão and G. N. Taranto, "Dynamic transmission capability calculation using integrated analysis tools and intelligent systems", IEEE Transactions on Power Systems, vol. 22, no. 4, pp 1760-1770, November 2007.

[26] F. B. Almeida, J. A. Passos Filho, J. L. R Pereira and R. M. Henriques, "Assessment of load modeling in power system security analysis based on static security regions," Journal of Control, Automation and Electrical Systems, vol. 24, no. 1, pp. 148-161, April 2013.

[27] H. Sarmiento, G. Pampin, R. Barajas, R. Castellanos, G. Villa and M. Mirabal, "Nomogram for assistance in voltage security visualization," Proceedings of the IEEE Power Systems Conference and Exposition, 2009.

[28] S. Kuenzel, "Modelling and control of an ACDC system with significant generation from wind,” Ph.D. Thesis, Imperial College London, 2014.

[29] P. Kundur, "Power System Stability and Control," McGrawHill, 1994.

[30] R. Adapa, "High-Wire Act: HVdc Technology: The State of the Art," IEEE Power and Energy Magazine, vol. 10, no. 6, pp.1829, November-December 2012.

[31] J. Qin and M. Saeedifard, "Reduced Switching-Frequency Voltage-Balancing Strategies for Modular Multilevel HVDC Converters," IEEE Transactions on Power Delivery, vol. 28, no. 4, pp. 2403-2410, October 2013.

[32] S. Kuenzel, L. P. Kunjumuhammed, B. C. Pal and I. Erlich, "Impact of wakes on wind farm inertial response," IEEE Transactions on Sustainable Energy,” vol. 5, no.1, pp. 237-245, January 2014.

[33] WECC Renewable Energy Modeling Task Force, "WECC wind power plant dynamic modeling guide,” April 2014. [Online]. Accessed November 2015. Available: https://goo.gl/wwLDzI.

\section{BIOGRAPHIES}

Tatiana Mariano Lessa Assis (S’02, GS'06, M’08, SM'12) received the D.Sc. degree in electrical engineering from the Federal University of Rio de Janeiro in 2007. Since 2011, Dr. Assis has been with the Electrical Engineering Department of Federal University of Rio de Janeiro, where she is an Associate Professor. Currently, she is on sabbatical leave as a Visiting Researcher at Imperial College London, UK.

Stefanie Kuenzel (GS'11, M'14) is Research Associate at the Control and Power Group in the Electrical and Electronic Engineering Department at Imperial College London, where she completed her Ph.D. on "Modelling and control of an ACDC system with significant generation from wind" between 2010 to 2014. Her current research interests include wind generator modelling and interaction studies.

Bikash Chandra Pal (M'00, SM'02, F'13) received the Ph.D. degree in electrical engineering from the Imperial College London in 1999. He is a Professor of Power Systems at Imperial College London and is research active in power system stability, control and computation. Prof Pal has graduated $18 \mathrm{PhDs}$ and published 65 technical papers in IEEE Transactions and IET journals. He is the Editor-in-Chief of IEEE Transactions on Sustainable Energy and Fellow of the IEEE. 